\title{
Conformal Transformations and Accelerated Cosmologies
}

\author{
James L. Crooks and Paul H. Frampton \\ University of North Carolina, Chapel Hill, NC 27599-3255, USA.
}

(Dated: September 20, 2018)

\begin{abstract}
A cosmological theory that predicts a late-time accelerated attractor with a constant dark matter to dark energy ratio can be said to solve the Coincidence Problem. Such cosmologies are naturally generated in the context of non-standard gravity theories under conformal transformation because of the resulting couplings between scalar fields and matter. The present work examines four classes of these transformed theories and finds that only a small subset-those with a single scalar field-are capable of solving the Coincidence Problem.
\end{abstract}




\section{INTRODUCTION}

The cosmological Coincidence Problem stems from the observation that the present-day ratio of dark matter (or total matter) to dark energy is near unity despite the fact that these two species are thought to evolve with time in different ways. Perhaps it is just a coincidence; however, physicists are weary of accepting it as such without investigating alternative explanations. One possibility is that the two species do not actually evolve indepentently. Indeed, a coupling between dark matter and dark energy can naturally yield a constant energy ratio near unity in the asymptotic time limit. If this steady-state is allowed and found to be consistent with a accelerated and dynamically stable universe then we can say that the theory generating it solves the Coincidence Problem.

Toward this end, a number of coupling schemes have been put forward (e.g. 1] and [2]). We will focus here on the scenario which involves conformally transforming a non-standard gravity theory. Generally, the alternative theory of gravity is taken to be the Brans-Dicke theory [3] (the simplest and best-motivated extension of general relativity relativity), in which Newton's constant is re-interpreted as a time-varying scalar field [4]. Amendola and various co-authors [5] 6], as well as other groups (e.g. 77] and 8]), have shown that the coupling derived from a Brans-Dicke theory yields a stable and accelerated critical point on which there is a constant ratio of dark matter to dark energy, thus solving the Coincidence Problem. However, many other gravity theories can be conformally transformed with differing results for the "Einstein frame" theory (see 9] for a review).

This article examines four classes of generalized gravity theories to find those that are incapable of solving the Coincidence Problem. It is shown that most kinds of non-standard gravity theories do not, upon conformal transformation, contain the accelerated, stable, and mixed critical points that are required. Lagrangians involving scalar fields coupled to terms quadratic in the Ricci scalar, as well as theories involving higher derivatives of the Ricci scalar, fail to solve the Coincidence Problem. On the other hand, both scalartensor theories linear in the Ricci scalar (like the Brans-Dicke theory) and nonlinear theories without scalar fields can contain the required critical points but only in special cases. The general requirements are that upon conformal transformation the theory must contain only

one scalar field, and that the dark matter-dark energy coupling function $\Upsilon(\phi)$ must be proportional to the square root of the scalar field coupling to gravity $\omega(\phi)$. 
Section II presents the method for conformally transforming a non-standard theory of gravitation. Sections IV through VII illustrate the practical use of conformal transformations by applying them to theories of four general types: linear scalar-tensor, nonlinear, quadratic scalar-tensor, and higher-order theories. The physical meaning of the conformal transformation is discussed in Section III]

A general consequence of conformal transformations is the appearance of a new coupling between a scalar field and the matter fields such that the stress-energy tensor of the matter fields is no longer conserved. Section VIII describes how the scalar field may be identified with dark energy. Particle physics experiments and astrophysical observations put severe limits on the non-conservation of normal baryonic matter, so Section IX] describes various ways to avoid these constraints. One way, devised by Damour et al. [4], is to couple the scalar field only to dark matter with an interaction mediated by the time-derivative of the scalar field multiplied by the energy density of dark matter. The modern version of this model is called the Coupled Quintessence Model. An alternative way introduced here also involves coupling a scalar field to dark matter, but now the interaction is mediated by baryons and non-relativistic neutrinos as well as well as dark matter itself.

Finally, to solve the Coincidence Problem we must determine which kinds of couplings allow the universe to converge to an accelerated, stable state containing a constant ratio of dark matter to dark energy. The first and last of these requirements reduce the space of possible schemes enormously. Sections $[\mathrm{X}$ and $\mathrm{XI}$ explain how a single scalar field model can meet these two requirements but a dual scalar field model cannot. Stability will not be discussed here as it is more dependent on the particular choices made for various arbitrary functions and parameter values. The general argument against two or more scalar fields is given in Section XII along with an explanation of why the single scalar field case is different. This section also shows that for a single scalar field to work there are constraints on the possible choices of functions for the scalar coupling to gravity, the dark matter-dark energy coupling, and the scalar field potential.

\section{GENERAL CONFORMAL TRANSFORMATIONS}

From a general action in a scalar-tensor or nonlinear gravity theory, 


$$
\widetilde{S}=\int d^{4} x\left\{\sqrt{-\widetilde{g}}\left[F(\psi, \widetilde{R})-A(\psi) \partial_{\mu} \psi \partial_{\nu} \psi \widetilde{g}^{\mu \nu}\right]-\widetilde{L}^{(M)}\right\},
$$

it is possible to generate the analogue of the Einstein field equations:

$$
\begin{aligned}
0= & \left(\frac{\partial F(\psi, \widetilde{R})}{\partial \widetilde{R}}\right)\left(\widetilde{R}_{\mu \nu}-\frac{1}{2} \widetilde{g}_{\mu \nu} \widetilde{R}\right)+\frac{1}{2} \widetilde{g}_{\mu \nu}\left(\frac{\partial F(\psi, \tilde{R})}{\partial \widetilde{R}} \widetilde{R}-F(\psi, \widetilde{R})\right) \\
& -A(\psi)\left(\partial_{\mu} \psi \partial_{\nu} \psi-\frac{1}{2} \widetilde{g}_{\mu \nu} \widetilde{g}^{\rho \sigma} \partial_{\rho} \psi \partial_{\sigma} \psi\right)+\widetilde{g}_{\mu \nu} \widetilde{\square}\left(\frac{\partial F(\psi, \widetilde{R})}{\partial \widetilde{R}}\right) \\
& -\widetilde{\nabla}_{\mu} \widetilde{\nabla}_{\nu}\left(\frac{\partial F(\psi, \widetilde{R})}{\partial \widetilde{R}}\right)-\frac{1}{2} \widetilde{T}_{\mu \nu}^{(M)} .
\end{aligned}
$$

The two terms involving second derivatives of $\partial F(\psi, \widetilde{R}) / \partial \widetilde{R}$ are due to the non-vanishing variation of the Riemann tensor in this general situation. Their derivation is given in [10].

Conformal transformations are transformations of the metric $\widetilde{g}_{\mu \nu}$ such that a nonstandard gravity theory may be written in a new form called the Einstein frame, the "frame" where the gravitational part of the action looks like the normal Einsteinian one (see [1]] for the original discussion of this general case).

To begin, define the conformal function $\Omega^{2}$ in terms of the Jordan frame gravitational action:

$$
\left|\frac{\partial F(\psi, \widetilde{R})}{\partial \widetilde{R}}\right| \equiv \frac{\Omega^{2}}{16 \pi G} .
$$

The conformal transformation (not a coordinate transformation in the usual sense) is then defined by the following transformation of the metric:

$$
\begin{aligned}
g_{\mu \nu} & =\Omega^{2} \widetilde{g}_{\mu \nu} \\
g^{\mu \nu} & =\Omega^{-2} \widetilde{g}^{\mu \nu} \\
\sqrt{-g} & =\Omega^{4} \sqrt{-\widetilde{g}}
\end{aligned}
$$

Because the gravitational part of the action is defined by the metric and its derivatives the geometrical side of the Einstein equations must transform as well: 


$$
\begin{aligned}
R_{\mu \nu}-\frac{1}{2} g_{\mu \nu} R= & \widetilde{R}_{\mu \nu}-\frac{1}{2} \widetilde{g}_{\mu \nu} \widetilde{R}-\Omega^{-2} \widetilde{\nabla}_{\mu} \widetilde{\nabla}_{\nu} \Omega^{2}+\Omega^{-2} \widetilde{g}_{\mu \nu} \widetilde{\square} \Omega^{2} \\
& +\frac{3}{2} \Omega^{-4}\left(\partial_{\mu} \Omega^{2} \partial_{\nu} \Omega^{2}-\frac{1}{2} \widetilde{g}_{\mu \nu} \widetilde{g}^{\alpha \beta} \partial_{\alpha} \Omega^{2} \partial_{\beta} \Omega^{2}\right) .
\end{aligned}
$$

The extra terms thus produced can be used to cancel the extra terms in equation (2). Let $\zeta=(\partial F(\psi, \widetilde{R}) / \partial \widetilde{R}) /|\partial F(\psi, \widetilde{R}) / \partial \widetilde{R}|$. Then, with the above substitution, equation (2) reads

$$
\begin{aligned}
R_{\mu \nu}-\frac{1}{2} g_{\mu \nu} R= & \zeta\left[8 \pi G \Omega^{-4} g_{\mu \nu}\left(F(\psi, \tilde{R})-\frac{\partial F(\psi, \widetilde{R})}{\partial \widetilde{R}} \widetilde{R}\right)+8 \pi G \Omega^{-2} \widetilde{T}_{\mu \nu}^{(M)}\right. \\
& \left.+16 \pi G \Omega^{-2} A(\psi)\left(\partial_{\mu} \psi \partial_{\nu} \psi-\frac{1}{2} g_{\mu \nu} g^{\rho \sigma} \partial_{\rho} \psi \partial_{\sigma} \psi\right)\right] \\
& +\frac{3}{2} \Omega^{-4}\left(\partial_{\mu} \Omega^{2} \partial_{\nu} \Omega^{2}-\frac{1}{2} g_{\mu \nu} g^{\alpha \beta} \partial_{\alpha} \Omega^{2} \partial_{\beta} \Omega^{2}\right) .
\end{aligned}
$$

In those cases where equation (3) may be written in the form $\widetilde{R}=\widetilde{R}\left(\Omega^{2}, \psi\right)$ it is possible to rewrite the second term on the right hand side of equation (8) as a scalar potential,

$$
\left.U\left(\psi, \Omega^{2}\right) \equiv \Omega^{-4}\left[\zeta F(\psi, \widetilde{R})-\left|\frac{\partial F(\psi, \widetilde{R})}{\partial \widetilde{R}}\right| \widetilde{R}\right]\right|_{\widetilde{R}=\widetilde{R}\left(\Omega^{2}, \psi\right)} .
$$

It is also possible to rewrite this term when equation (3) does not involve $\widetilde{R}$, i.e. when $F(\widetilde{R}, \psi)$ is linear in $\widetilde{R}$. Then $U\left(\psi, \Omega^{2}\right)$ is simply proportional to the scalar potential term in $F(\widetilde{R}, \psi)$ if such a term exists, and zero otherwise.

Since the Einstein equations should by definition have the normal form in the Einstein frame, equation (8) implies

$$
\begin{aligned}
T_{\mu \nu}^{\left(\psi, \Omega^{2}\right)} \equiv & 2 \zeta A(\psi) \Omega^{-2}\left(\partial_{\mu} \psi \partial_{\nu} \psi-\frac{1}{2} g_{\mu \nu} g^{\rho \sigma} \partial_{\rho} \psi \partial_{\sigma} \psi\right)+g_{\mu \nu} U\left(\psi, \Omega^{2}\right) \\
& +\frac{3}{16 \pi G} \Omega^{-4}\left(\partial_{\mu} \Omega^{2} \partial_{\nu} \Omega^{2}-\frac{1}{2} g_{\mu \nu} g^{\alpha \beta} \partial_{\alpha} \Omega^{2} \partial_{\beta} \Omega^{2}\right)
\end{aligned}
$$

and

$$
T_{\mu \nu}^{(M)} \equiv \zeta \Omega^{-2} \widetilde{T}_{\mu \nu}^{(M)}
$$

With these definitions equation (8) then reads 


$$
R_{\mu \nu}-\frac{1}{2} g_{\mu \nu} R=8 \pi G\left(T_{\mu \nu}^{(M)}+T_{\mu \nu}^{\left(\psi, \Omega^{2}\right)}\right) .
$$

However, since the relation

$$
\widehat{T}_{\mu \nu}=\frac{2}{\sqrt{-\widehat{g}}} \frac{\delta \widehat{L}}{\delta \widehat{g}^{\mu \nu}}
$$

must be valid in either frame, we can reverse-engineer equation (10) to get an expression for the Lagrangian in the Einstein frame,

$$
\begin{aligned}
L^{\left(\psi, \Omega^{2}\right)}= & \sqrt{-g}\left[\zeta A(\psi) \Omega^{-2} g^{\mu \nu} \partial_{\mu} \psi \partial_{\nu} \psi-U\left(\psi, \Omega^{2}\right)\right. \\
& \left.+\frac{3}{32 \pi G} \Omega^{-4} g^{\mu \nu} \partial_{\mu} \Omega^{2} \partial_{\nu} \Omega^{2}\right] .
\end{aligned}
$$

Then with this identity we can write down the Einstein frame action that generates equation (12),

$$
S=\int d^{4} x\left\{\frac{\sqrt{-g} R}{16 \pi G}-L^{\left(\psi, \Omega^{2}\right)}-L^{(M)}\right\} .
$$

An important property of matter fields under conformal transformation is that a stressenergy tensor conserved in the Jordan frame is not generally conserved in the Einstein frame and vice-versa. To see this, first expand the 4-divergence of the stress-energy tensor of matter species $\mathrm{X}$ in the Jordan frame and then substitute appropriate factors of $\Omega^{2}$ 12]:

$$
\begin{aligned}
\widetilde{\nabla}_{\mu} \widetilde{T}_{\nu}^{\mu(X)} & =\frac{1}{\sqrt{-\widetilde{g}}} \partial_{\mu}\left(\sqrt{-\widetilde{g}} \widetilde{T}_{\nu}^{\mu(X)}\right)-\frac{1}{2} \widetilde{T}^{\mu \gamma(X)} \partial_{\nu} \widetilde{g}_{\mu \gamma} \\
& =\frac{\Omega^{4}}{\sqrt{-g}} \partial_{\mu}\left(\sqrt{-g} T_{\nu}^{\mu(X)}\right)-\frac{1}{2} \Omega^{6} T^{\mu \gamma(X)} \partial_{\nu}\left(\Omega^{-2} g_{\mu \gamma}\right) \\
& =\Omega^{4} \nabla_{\mu} T_{\nu}^{\mu(X)}+\frac{1}{2} \Omega^{2} \partial_{\nu} \Omega^{2} T^{(X)} \\
\nabla_{\mu} T_{\nu}^{\mu(X)} & =\Omega^{-4} \widetilde{\nabla}_{\nu} \widetilde{T}_{\nu}^{\mu(X)}-\frac{1}{2} \Omega^{-6} \partial_{\nu} \Omega^{2} \widetilde{T}^{(X)}
\end{aligned}
$$

Therefore, when the stress-energy tensor is conserved in one frame it is not conserved in the other except if the species $\mathrm{X}$ is radiation $\left(\widehat{T}^{(R)}=0\right)$ or $\Omega^{2}$ is constant.

To see this another way, vary the action to get the equations of motion in the Einstein frame. Varying equation (15) with respect to $\psi$ generates the $\psi$ equation of motion 


$$
\begin{aligned}
2 \zeta A(\psi) \Omega^{-2} \square \psi= & 2 \zeta A(\psi) \Omega^{-4} g^{\mu \nu} \partial_{\mu} \Omega^{2} \partial_{\nu} \psi-\zeta \frac{\partial A(\psi)}{\partial \psi} \Omega^{-2} g^{\mu \nu} \partial_{\mu} \psi \partial_{\nu} \psi \\
& -\frac{\partial U\left(\psi, \Omega^{2}\right)}{\partial \psi} .
\end{aligned}
$$

Varying the action with respect to $\Omega^{2}$ (using the trace of equation (17) to transform between frames) yields the equation of motion for $\Omega^{2}$. Because of the transformation between frames this equation includes a Ricci scalar term which may be eliminated by substituting the trace of equation (8). Doing so yields

$$
\begin{aligned}
\square \Omega^{2}= & \Omega^{-2} g^{\mu \nu} \partial_{\mu} \Omega^{2} \partial_{\nu} \Omega^{2}-\frac{16 \pi G}{3} \zeta A(\psi) g^{\mu \nu} \partial_{\mu} \psi \partial_{\nu} \psi \\
& -\frac{16 \pi G}{3} \Omega^{4} \frac{\partial U\left(\psi, \Omega^{2}\right)}{\partial \Omega^{2}}+\frac{8 \pi G}{3} \Omega^{2} T^{(M)} .
\end{aligned}
$$

Finally, we take the 4-divergence of equation (12) and substitute in the equations of motion. After cancellation the only terms remaining give

$$
\nabla^{\mu} T_{\mu \nu}^{(M)}=-\frac{1}{2} \Omega^{-2} \partial_{\nu} \Omega^{2} T^{(M)} .
$$

However, since the 4-divergence must evaluate to zero on both sides of the Einstein equations (equation (8)) we can read off:

$$
\nabla^{\mu} T_{\mu \nu}^{\left(\psi, \Omega^{2}\right)}=\frac{1}{2} \Omega^{-2} \partial_{\nu} \Omega^{2} T^{(M)} .
$$

Note that this does not contradict equation (16) since $\psi$ and $\Omega^{2}$ are not "matter fields" in the sense used above, i.e., they do not appear in the matter part of the original action.

\section{INTERPRETATION}

What is the physical significance of a conformal transformation? What is the relationship between the Jordan frame and the Einstein frame? If we assume that only the Einstein frame is directly observable, is the Jordan frame anything more than a mathematical device? There is much debate surrounding these questions (see, e.g., [13], [14], and [15]). Different authors

interpret conformal transformations in different ways; the debate often revolves around the question: which of the frames is "physical"? 
Some simply define the "physical" frame as the one in which the matter stress-energy tensor is conserved. Others define it as the one in which we happen to be making observations, which is not necessarily the same thing. Neither position can be rejected out of hand. It seems that either frame may be pressed into service as the "physical" frame depending on the use to which the theory is being put. Furthermore, even once the "physical" frame is chosen, the meaning of the other frame is not obvious. Is it just a convenient fiction? Is it possible for both frames to be physically meaningful?

Perhaps the most galling problem with conformal transformations theories is that often no interpretation-or even agnosticism-is stated explicitly. The interpretation given in the present work depends on the way in which the interaction term is partitioned among the different matter species, as will be discussed in Section [X] For now we will dodge the question and look at various classes of non-standard gravity theories and what happens to them upon conformal transformation.

\section{LINEAR SCALAR-TENSOR THEORIES}

Linear scalar-tensor theories are those for which $F(\psi, \widetilde{R})$ in equation (11) takes the form $F(\psi, \widetilde{R})=f(\psi) \widetilde{R}+V(\psi)[16]$. In this case $\zeta$ takes the form $\zeta=|f(\psi)| / f(\psi)$.

Since these theories are linear in $\widetilde{R}$ it is obvious that $\Omega^{2}$ is a function only of $\psi$. This has two consequences. First, if $V(\psi)$ exists then

$$
U\left(\psi, \Omega^{2}\right)=V(\psi) \frac{|f(\psi)|}{f^{3}(\psi)} \text { when } f(\psi) \neq 0,
$$

though presumably $V(\psi)$ could be chosen such that the ratio on the right hand side is defined when $f(\psi)=0$. Second, there is effectively only a single scalar field in the action since the two kinetic terms can be directly added together. Going back to equation (14) we can therefore make the substitution

$$
\phi=\int d \psi \sqrt{\frac{3}{16 \pi G} \Omega^{-4}(\psi)\left(\frac{\partial \Omega^{2}(\psi)}{\partial \psi}\right)^{2}+2 \zeta A(\psi) \Omega^{-2}(\psi)}
$$

to get a single minimally coupled scalar field in the action. Now instead of two equations of motion there is only one, 


$$
\square \phi+\frac{\partial U(\phi)}{\partial \phi}=\frac{1}{2} \Omega^{-2}(\phi) \frac{d \Omega^{2}(\phi)}{d \phi} T^{(M)} .
$$

Of course we could also set up equation (22) such that the resulting action would contain a non-minimally coupled scalar field; the choice is arbitrary, though the minimally coupled option is favored on aesthetic grounds.

Equation (22) also implies that when $f(\psi) \propto \psi^{n}$ and $A(\psi) \propto \psi^{n-2}$ for a given $n$ the resulting Einstein frame equations of motion are indistinguishable from the Brans-Dicke $(n=1)$ case. The same applies if both $f(\psi)$ and $A(\psi)$ are proportional to $e^{\mu \psi}$, or in any other circumstance in which $A(\psi) \propto \frac{1}{f(\psi)}\left(\frac{\partial f(\psi)}{\partial \psi}\right)^{2}$ holds. Curiously, this indistinguishability means that if the Jordan frame were inferred to be the natural one for the dark sector there would be no way to distinguish one such theory from any other since they are all equivalent in the Einstein frame.

\section{NONLINEAR GRAVITY THEORIES}

Nonlinear gravity theories are those for which $F(\psi, \widetilde{R})$ do not depend on $\psi$, i.e., $F(\psi, \widetilde{R})=$ $F(\widetilde{R})[15]$. Since there are no scalar fields coupled to gravity in the Jordan frame we can ignore $A(\psi)$ and $V(\psi)$. This means that the resulting Einstein frame potential depends entirely on the Jordan frame theory chosen-there is no freedom to choose the functional form. The Einstein frame equations of motion, though, are of exactly the same form as those in the linear scalar-tensor case given by equation (23) once $\Omega^{2}$ has been transformed into the minimally coupled field $\phi$ using

$$
\Omega^{2}=e^{\sqrt{\frac{16 \pi G}{3}} \phi} .
$$

However, because $\Omega^{2}$ can often be defined as a function of $\widetilde{R}$ it is sometimes possible to relate $\widetilde{R}$ directly to $\phi$. The absolute value in equation (3) complicates the matter since two values of $\widetilde{R}$ can correspond to any one value of $\Omega^{2}$. In practice, however, one of the two $\zeta$ values may not be available since $F(\widetilde{R})$ may be assumed real and $F^{\prime}(\widetilde{R})$ is sometimes intrinsically positive or negative. On the other hand, even if both values are available we are effectively free to choose which one we want since the value of $\widetilde{R}$ is not observable anyway.

In addition to $\zeta$, however, there is another sign ambiguity when $F^{\prime}(\widetilde{R})$ is an even function of $\widetilde{R}$. For example when $\mathrm{f} F(\widetilde{R}) \propto \widetilde{R}\left(1+(\alpha \widetilde{R})^{-2}\right)$ there are four values of $\widetilde{R}$ corresponding 
to each negative value of $\phi$; specifically, there is a splitting due to $\zeta$ and another due to the symmetry of $F^{\prime}(\widetilde{R})$, for a total of four solutions.

It is interesting to compare these theories to the cubic one: $\left(F(\widetilde{R}) \propto \widetilde{R}+\alpha \widetilde{R}^{2}+\gamma \widetilde{R}^{3}\right)$. When $\alpha^{2} / \beta>3$ the cubic theory also has four possible values of $\widetilde{R}$ for certain values of $\phi$. However, they are not symmetrically distributed and therefore the corresponding potentials cannot in general be written in a simple form involving $\zeta$ and \pm . The problem of having too many Einstein-frame potentials obviously gets worse with higher-order polynomials.

Also, despite the fact that each Jordan frame theory corresponds to a unique potential (modulo possible sign ambiguities) in the Einstein frame, there are theories outside this class (e.g., linear scalar-tensor theories) whose equation of motion is the same and whose potentials are completely arbitrary. Thus there is no way to distinguish any Jordan-frame theory in this class from a scalar-tensor theory using only the Einstein-frame potential.

\section{QUADRATIC SCALAR-TENSOR}

Quadratic scalar-tensor theories are those for which $F(\psi, \widetilde{R})$ takes the form

$$
F(\psi, \widetilde{R})=f(\psi) \widetilde{R}+g(\psi) \widetilde{R}^{2}+V(\psi)
$$

The two best known models in this class are the dilaton [17] and renormalizable [18] theories. Unlike the nonlinear gravity and linear scalar-tensor cases, $\Omega^{2}$ here involves both a function

of $\psi$ and a nonlinear power of $\widetilde{R}$. This means we must keep both kinetic terms in equation (10); we can neither combine them as in the nonlinear gravity examples nor eliminate one as in the linear scalar-tensor examples. However, we can transform $\Omega^{2}$ into $\phi$ as before to make at least one of the kinetic terms minimally coupled:

$$
\begin{aligned}
T_{\mu \nu}^{(\psi, \phi)} \equiv & 2 \zeta A(\psi) e^{-\sqrt{\frac{16 \pi G}{3}} \phi}\left(\partial_{\mu} \psi \partial_{\nu} \psi-\frac{1}{2} g_{\mu \nu} g^{\rho \sigma} \partial_{\rho} \psi \partial_{\sigma} \psi\right)+g_{\mu \nu} U(\psi, \phi) \\
& +\partial_{\mu} \phi \partial_{\nu} \phi-\frac{1}{2} g_{\mu \nu} g^{\alpha \beta} \partial_{\alpha} \phi \partial_{\beta} \phi
\end{aligned}
$$

This leads to a potential,

$$
U(\psi, \phi)=\zeta V(\psi) e^{-2 \sqrt{\frac{16 \pi G}{3}} \phi}-\frac{\zeta}{4 g(\psi)}\left[\zeta f(\psi) e^{-\sqrt{\frac{16 \pi G}{3}} \phi}-\frac{1}{16 \pi G}\right]^{2}
$$


an equation of motion for $\psi$,

$$
\begin{aligned}
\square \psi+\frac{\zeta}{2 A(\psi)} e^{\sqrt{\frac{16 \pi G}{3}} \phi} \frac{\partial U(\psi, \phi)}{\partial \psi}= & -\frac{1}{2} \frac{\partial \log A(\psi)}{\partial \psi} e^{-\sqrt{\frac{16 \pi G}{3}} \phi} g^{\mu \nu} \partial_{\mu} \psi \partial_{\nu} \psi \\
& +\sqrt{\frac{16 \pi G}{3}} g^{\mu \nu} \partial_{\mu} \psi \partial_{\nu} \phi
\end{aligned}
$$

and an equation of motion for $\phi$,

$$
\square \phi+\frac{\partial U(\psi, \phi)}{\partial \phi}=-\zeta \sqrt{\frac{16 \pi G}{3}} A(\psi) e^{-\sqrt{\frac{16 \pi G}{3}} \phi} g^{\mu \nu} \partial_{\mu} \psi \partial_{\nu} \psi+\frac{1}{2} \sqrt{\frac{16 \pi G}{3}} T^{(M)}
$$

These theories do not share the distinguishability problem of the previous two types since there are two coupled scalar fields with non-trivial equations of motion and a potential determined uniquely (modulo $\zeta$ ) by the specific Jordan-frame theory. It would be quite bizarre for arbitrary functions in some other Jordan-frame theory to reproduce these equations.

\section{HIGHER-ORDER GRAVITY THEORIES}

There are many gravitational actions without scalar fields we have thus far neglected. For example we have looked only at theories involving the Ricci scalar and have ignored the Ricci and Riemann tensors. It is possible to imagine theories with terms in the action proportional to $\widetilde{R}_{\mu \nu \rho \sigma} \widetilde{R}^{\mu \nu \rho \sigma}$ or to $\widetilde{R}_{\mu \nu} \widetilde{R}^{\mu \nu}$. However, the former may be eliminated as part of a total divergence and the latter is reducible to a form involving only $\widetilde{R}^{2}$ in a FriedmannRobertson-Walker metric [19] as will be assumed throughout this paper.

Alternatively, we could look at actions involving functions of derivatives of the Ricci scalar: $F\left(\widetilde{R}, \widetilde{\square} \widetilde{R}, \ldots, \widetilde{\square}^{n} \widetilde{R}\right)$ (see [19], [20], and [14]). Wands [19] has shown that, by a redefinition of variables (not, it should be emphasized, a conformal transformation) $\psi_{i} \equiv$ $\widetilde{\square}^{i} \widetilde{R}$, we may write this general action as

$$
\widetilde{S}=\int d^{4} x\left\{\sqrt{-\widetilde{g}}\left[\left(\sum_{j=0}^{n} \widetilde{\square}^{j} \frac{\partial F}{\partial \psi_{j}}\right) \widetilde{R}+F-\sum_{j=0}^{n} \psi_{j} \frac{\partial F}{\partial \psi_{j}}\right]-\widetilde{L}^{(M)}\right\}
$$

where $F$ is now written as $F\left(\psi_{0}, \ldots, \psi_{n}\right)$. Thus a higher-order gravity theory may be rewritten in a form similar to that of a linear or quadratic scalar-tensor theory. In fact, this 
similarity is quite deep. Wands gives the example $F(\widetilde{R}, \widetilde{\square} \widetilde{R}) \propto \widetilde{R}+\gamma \widetilde{R} \widetilde{\square} \widetilde{R}$ and shows that under conformal transformation it has a Lagrangian of the form given by equation (14). Redefining $\Omega^{2}=\exp \left(\sqrt{\frac{16 \pi G}{3}} \phi\right)$ and $\psi_{0}=\sqrt{\frac{8 \pi G}{\gamma}} \psi$ the stress-energy tensor is then just equation (26) with $\zeta A(\psi)=\frac{1}{2}$. Furthermore, the form of the potential is uniquely determined to be

$$
U(\psi, \phi)=\frac{\psi}{\sqrt{32 \pi G \gamma}}\left(e^{-\sqrt{\frac{16 \pi G}{3}} \phi}-e^{2 \sqrt{\frac{16 \pi G}{3}} \phi}\right) .
$$

Where does the kinetic term for $\psi_{0}$ (or equivalently $\psi$ ) come from? When we replace $\psi_{1}$ with $\Omega^{2}$ a new term proportional to $\psi_{0} \widetilde{\square} \psi_{0}$ appears in the action, which, after integration by parts, becomes a kinetic term for $\psi_{0}$.

What about the general case? If we take the conformal function to be

$$
\Omega^{2}=\sum_{j=0}^{n} \widetilde{\square}^{j} \frac{\partial F}{\partial \psi_{j}}
$$

and convert to the Einstein frame the result depends strongly on the number of derivatives of the Ricci scalar. Quadratic or higher polynomials in $\widetilde{\square} \widetilde{R}$ yield three or more scalar fields, while terms of order $\widetilde{\square}^{2} \widetilde{R}, \widetilde{\square}^{3} \widetilde{R}$, etc..., yield more than two scalar fields and/or kinetic terms involving higher derivatives. The most general form of higher-order gravity theory that has an Einstein frame Lagrangian equivalent to equation (14) appears to be

$$
F(\widetilde{R}, \widetilde{\square} \widetilde{R})=f_{0}(\widetilde{R})+f_{1}(\widetilde{R}) \widetilde{\square} \widetilde{R}
$$

The Einstein frame version of the theory has the potential

$$
U\left(\psi_{0}, \Omega^{2}\right)=\Omega^{-4}\left(f_{1}\left(\psi_{0}\right)-\Omega^{2} \psi_{0}\right)
$$

and we may identify

$$
\zeta A\left(\psi_{0}\right)=\frac{\partial f_{1}\left(\psi_{0}\right)}{\partial \psi_{0}}
$$

By contrast, a theory linear in $\widetilde{\square}^{2} \widetilde{R}$ instead of just $\widetilde{\square} \widetilde{R}$,

$$
F\left(\widetilde{R}, \widetilde{\square} \widetilde{R}, \widetilde{\square}^{2} \widetilde{R}\right)=f_{0}(\widetilde{R})+f_{1}(\widetilde{R}) g(\widetilde{\square} \widetilde{R})+f_{2}(\widetilde{R}) \widetilde{\square}^{2} \widetilde{R}
$$


cannot be written in a form equivalent to equation (14). In this case we find that the second and third terms under the brackets in equation (30), after replacing $\psi_{2}$ with $\Omega^{2}$, may be written as

$$
\begin{aligned}
F-\sum_{j=0}^{2} \psi_{j} \frac{\partial F}{\partial \psi_{j}}= & f_{0}\left(\psi_{0}\right)+f_{1}\left(\psi_{0}\right) g\left(\psi_{1}\right)-f_{1}\left(\psi_{0}\right) \frac{d g\left(\psi_{1}\right)}{d \psi_{1}} \psi_{1}+\frac{d f_{2}\left(\psi_{0}\right)}{d \psi_{0}} \psi_{1}^{2} \\
& -\psi_{0} \Omega^{2}+\left(\frac{d^{2} f_{2}\left(\psi_{0}\right)}{d \psi_{0}^{2}} \psi_{1}-\frac{d f_{1}\left(\psi_{0}\right)}{d \psi_{0}} \frac{d g\left(\psi_{1}\right)}{d \psi_{1}}\right) \widetilde{g}^{\mu \nu} \partial_{\mu} \psi_{0} \partial_{\nu} \psi_{0} \\
& -f_{1}\left(\psi_{0}\right) \frac{d^{2} g\left(\psi_{1}\right)}{d \psi_{1}^{2}} \widetilde{g}^{\mu \nu} \partial_{\mu} \psi_{0} \partial_{\nu} \psi_{1}
\end{aligned}
$$

There are now three different kinetic terms in the total Einstein-frame action (the third being the one for $\Omega^{2}$ produced by the conformal transformation). This is the price that is paid for having an unusual Jordan-frame action.

\section{RELEVANCE TO COSMOLOGY}

The purpose of describing all of these non-standard gravity theories and their conformal transformations is to help explain dark energy and the coincidence problem. To do so we must identify the scalar field or fields with dark energy. The standard scalar field dark energy model (without a conformal transformation) is called Quintessence 21], so we are just assigning the name "quintessence" to all the scalar fields in any particular model. In this way we may use the scalar-matter couplings generated by conformal transformation to produce a constant ratio of matter to dark energy and thus to solve the coincidence problem.

\section{MATTER-DARK ENERGY COUPLING}

If we try to model the universe with a stress-energy conservation equation like equation (19) we quickly run into problems due to the fact that $\partial_{\nu} \log \Omega^{2}$ must be small for the entire history of the universe if cosmological observations and tests of general relativity are to be believed. Simply put, we know that baryons, neutrinos, and photons have not had cosmologically significant interactions with other species. Before decoupling, their interactions

appear to be consistent with the Standard Model of particle physics, and after decoupling their stress-energy tensors are independently conserved to good approximation. If we look 
at a linear scalar-tensor example with $\frac{1}{2} \frac{d \log \Omega^{2}(\phi)}{d \phi}=\xi \sqrt{\frac{16 \pi G}{3}}$ the parameter $|\xi| \sqrt{\frac{16 \pi G}{3}}$ must be less than 0.032 to be consistent with radar delay experiments [4, 22].

The problem for conformally transformed theories does not anyway lie with photons and relativistic neutrinos since their stress-energy tensors are trace-free; the problem instead lies with cold dark matter, baryons, and non-relativistic neutrinos. We can state this problem as follows: how do we partition

$$
\nabla^{\mu}\left(T_{\mu \nu}^{B}+T_{\mu \nu}^{C}+T_{\mu \nu}^{\nu, n r}\right)=-\frac{1}{2} \Omega^{-2} \partial_{\nu} \Omega^{2}\left(T^{B}+T^{C}+T^{\nu, n r}\right)
$$

such that the resulting cosmological model is distinct from the standard one and yet is consistent with the observational bounds on couplings to visible matter?

One possibility is for each species to couple only to itself:

$$
\begin{aligned}
\nabla^{\mu} T_{\mu \nu}^{C} & =-\frac{1}{2} \Omega^{-2} \partial_{\nu} \Omega^{2} T^{C} \\
\nabla^{\mu} T_{\mu \nu}^{B} & =-\frac{1}{2} \Omega^{-2} \partial_{\nu} \Omega^{2} T^{B} \\
\nabla^{\mu} T_{\mu \nu}^{\nu, n r} & =-\frac{1}{2} \Omega^{-2} \partial_{\nu} \Omega^{2} T^{\nu, n r} .
\end{aligned}
$$

However, since the constraints from the visible sector on $\Omega^{-2} \partial_{\nu} \Omega^{2}$ also constrain the dark sector this model is not observationally distinct from the standard cosmology. An oft-used alternative due to Damour et al. [4] is to postulate that $\Omega^{-2} \partial_{\nu} \Omega^{2}$ from the latter two equations is different from that of the first. This allows coupling to the dark sector to be less constrained than coupling to the visible sector. It is on this utilitarian basis that the modification (usually with $\Omega^{-2} \partial_{\nu} \Omega^{2}=0$ in the visible sector) is used in cosmology as the Coupled Quintessence Model [23].

It is important to stress that the coupling asymmetry has important effects; physically it means that dark matter and visible matter naturally inhabit two different gravitational theories, where the "natural" theory for a given matter species is here taken to mean the one in which its stress-energy tensor is conserved. Thus the equivalence principle is violated in this model, a not inconsiderable loss. We are also left with a deeper question of how we interpret the conformal transformation that gave rise to these matter couplings. What is the relationship between the Jordan and Einstein frame theories now? Breaking the equivalence principle actually puts the two frames on more footing since each has its own "natural" matter fields. From the point of view of conserving matter stress-energy they are 
equally valid. Thus, it seems that the mathematical inconsistency results in an appealing interpretation in that both frames can be said to be physically relevant.

Another possibility is to have cold dark matter couple to all three species, leaving the baryons and non-relativistic neutrinos to be exactly conserved:

$$
\begin{aligned}
\nabla^{\mu} T_{\mu \nu}^{C} & =-\frac{1}{2} \Omega^{-2} \partial_{\nu} \Omega^{2}\left(T^{B}+T^{C}+T^{\nu, n r}\right) \\
\nabla^{\mu} T_{\mu \nu}^{B} & =0 \\
\nabla^{\mu} T_{\mu \nu}^{\nu, n r} & =0 .
\end{aligned}
$$

Here the evolution of the visible components is affected by the dark sector only through the Friedmann equation, but the interaction between dark matter and dark energy is catalyzed by the presence of baryons and non-relativistic neutrinos. Let us refer to this as the Catalyzed Quintessence model. There is nothing mathematically inconsistent with this possibility though it may well be physically questionable as it allows the dark matter energy density to go negative in the Einstein frame, though total energy is still conserved. Although this is certainly not a desirable feature, the model may be considered as a sort of matter analogue to phantom energy 24]. The application of this model to cosmology is discussed further in [25].

What is the status of the two frames in this model? Oddly, the stress-energy tensor of visible matter is conserved in both frames. This does not mean that all observations will be the same in both frames since the influence of gravity will in general be quite different, and furthermore dark matter will be conserved in one frame and not in the other. Thus there is no simple answer to the question, "Which frame is the physical one?" The criterion of stress-energy conservation may not give an unambiguous answer. Additionally, there is no symmetry here between the two frames, and indeed the Jordan frame is arguably the more natural one since all types of matter are conserved in it. Thus the natural frame and the observable frame need not be the same; we have to define the observable frame to be physical a posteriori. 


\section{ASYMPTOTIC BEHAVIOR: SINGLE SCALAR FIELD}

Since we are trying to explain the coincidence problem it suffices to look for theories whose dynamics contain a stable and accelerated critical point where the ratio of dark matter to dark energy is constant. Thus far the theories presented have generally contained arbitrary functions, usually in the form of the Jordan frame potential, that must be chosen before their dynamics can be determined. There are infinitely many possible choices of potential, but it turns out that it is not necessary to search them all to find one with a mixed critical point; instead we can work backwards from the requirement of a mixed critical point and the equations of motion to construct the form that works. The requirement of acceleration can then be imposed. The requirement of stability will not be discussed here as it depends on the specific parameter values.

For a linear scalar-tensor or nonlinear gravity system in an expanding FriedmannRobertson-Walker (FRW) universe the scalar field equation of motion in the Einstein frame is

$$
\omega\left(\ddot{\phi}+3 \frac{\dot{a}}{a} \dot{\phi}\right)+\frac{d U(\phi)}{d \phi}=\Upsilon(\phi) \rho_{M},
$$

while the dark matter energy conservation equation (23) is

$$
\dot{\rho}_{M}+3 \frac{\dot{a}}{a} \rho_{M}=-\Upsilon(\phi) \rho_{M} \dot{\phi}
$$

where $\Upsilon(\phi) \equiv \frac{1}{2} \Omega^{-2}(\phi) \frac{d \Omega^{2}(\phi)}{d \phi}$.

On the critical point we require the potential, kinetic, and dark matter energy densities to be exactly proportional:

$$
\rho_{M}=\epsilon U(\phi)=\kappa \frac{\omega}{2} \dot{\phi}^{2}
$$

where $\epsilon$ and $\kappa$ are positive constants. Using the Friedmann equation on the attractor,

$$
\left(\frac{\dot{a}}{a}\right)^{2}=\frac{8 \pi G}{3}\left(\frac{\omega}{2} \dot{\phi}^{2}+V(\phi)+\rho_{M}\right),
$$

the conservation equation for $\rho_{M}$, the equation of motion for $\phi$, and the above proportionality constraints it is possible to show that the coupled energy densities all evolve with the scale factor like 


$$
\rho \sim a^{-\frac{3 \epsilon(\kappa+2)}{\epsilon+\kappa+\epsilon \kappa}}
$$

Specifically, $\rho_{M}$ has the solution

$$
\rho_{M}=\left(\frac{a}{a_{0}}\right)^{-\frac{3 \epsilon(\kappa+2)}{\epsilon+\kappa+\epsilon \kappa}} \rho_{M, 0},
$$

where subscript $M$ denotes either dark matter alone or the combination of all matter species depending on which model is chosen. On the other hand, the scale factor evolves with time like

$$
a(t)=a_{0}\left(\frac{t}{t_{0}}\right)^{\frac{2(\epsilon+\kappa+\epsilon \kappa)}{3 \epsilon(2+\kappa)}}
$$

so that at late time the coupled energy densities evolve like $\rho \sim t^{-2}$.

From this we may determine the time evolution of $\phi$, which turns out to be

$$
\phi=\phi_{0}+\frac{1}{\sqrt{\pi G \omega}} \ln \left[\left(\frac{t}{t_{0}}\right)^{ \pm \frac{\sqrt{\epsilon+\kappa+\epsilon \kappa}}{(2+\kappa) \sqrt{3 \epsilon}}}\right] .
$$

With the time evolution and the coupling ratios it is then possible to calculate the exact form that the potential must have for there to be a mixed attractor:

$$
U(\phi)=\frac{\rho_{M, 0}}{\epsilon} e^{ \pm(2+\kappa) \sqrt{\frac{12 \omega \epsilon \pi G}{\epsilon+\kappa+\epsilon \kappa}}\left(\phi-\phi_{0}\right)},
$$

where

$$
\rho_{M, 0}=\frac{\kappa(\epsilon+\kappa+\epsilon \kappa)}{6 \pi G \epsilon(2+\kappa)^{2} t_{0}^{2}}
$$

If we let $U(\phi) \sim e^{\mu \sqrt{\frac{16 \pi G}{3}} \phi}$ and compare this to the required form for a critical point to exist we find that the model parameter $\mu$ is related to the energy density ratios by

$$
\frac{\mu}{\sqrt{\omega}}= \pm \frac{3 \sqrt{\epsilon}(2+\kappa)}{2 \sqrt{\epsilon+\kappa+\epsilon \kappa}}
$$

Furthermore, if we look at the simplest model of interest, that for which $\Upsilon(\phi)=\xi \sqrt{\frac{16 \pi G}{3}}$, we can use our knowledge of $\rho_{M}(t)$ and $\phi(t)$ to simplify the dark matter conservation equation. The latter becomes 


$$
\frac{\kappa(\epsilon+\kappa+\epsilon \kappa)\left(3 \kappa-3 \epsilon \mp 2 \frac{\xi}{\sqrt{\omega}} \sqrt{\epsilon(\epsilon+\kappa+\epsilon \kappa)}\right)}{6 \epsilon^{2}(2+\kappa)}=0 .
$$

Since we are assuming $\epsilon$ and $\gamma$ are positive, this constraint gives us the following relation between the model parameter $\xi$ and the coupling ratios:

$$
\frac{\xi}{\sqrt{\omega}}=\mp \frac{3(\epsilon-\kappa)}{2 \sqrt{\epsilon(\epsilon+\kappa+\epsilon \kappa)}} .
$$

Therefore, given $\omega, \mu$, and $\xi$ we can write down exactly what the various energy densities (written here as fractions of the critical density) will be at the mixed critical point:

$$
\begin{aligned}
\Omega_{M} & =\frac{2 \mu^{2}+2 \mu \xi-9 \omega}{2(\mu+\xi)^{2}} \\
\Omega_{U} & =\frac{4 \mu \xi+4 \xi^{2}+9 \omega}{4(\mu+\xi)^{2}} \\
\Omega_{K} & =\frac{9 \omega}{4(\mu+\xi)^{2}} .
\end{aligned}
$$

Looking ahead, it is possible to find the region of the parameter space (the model parameters) in which the critical point is accelerated by determining where the overall equation of state,

$$
w=\frac{\Omega_{K}-\Omega_{U}}{\Omega_{K}+\Omega_{U}+\Omega_{M}}=\frac{\epsilon-\kappa}{\epsilon+\kappa+\epsilon \kappa},
$$

is less than $-\frac{1}{3}$. Such a region does in fact exist as was shown in [25].

\section{ASYMPTOTIC BEHAVIOR: DUAL SCALAR FIELDS}

The quadratic scalar-tensor case is somewhat more complicated. In an expanding homogeneous isotropic universe the equations (28) and (29) take the form :

$$
\begin{aligned}
\omega\left(\ddot{\phi}+3 \frac{\dot{a}}{a} \dot{\phi}\right)= & -\frac{\partial U(\psi, \phi)}{\partial \phi}+\sqrt{\frac{4 \pi G \omega}{3}}\left(\rho_{M}-2 e^{-\sqrt{\frac{16 \pi G \omega}{3}} \phi} \zeta(\psi) \dot{\psi}^{2}\right) \\
\ddot{\psi}+3 \frac{\dot{a}}{a} \dot{\psi}= & -e^{\sqrt{\frac{16 \pi G \omega}{3}} \phi} \frac{\zeta}{2 A(\psi)} \frac{\partial U(\psi, \phi)}{\partial \psi} \\
& -\frac{1}{2} \frac{d \log A(\psi)}{d \psi} \dot{\psi}^{2}+2 \sqrt{\frac{4 \pi G \omega}{3}} \dot{\phi} \dot{\psi},
\end{aligned}
$$


while equation (19) reduces to

$$
\dot{\rho}_{M}+3 \frac{\dot{a}}{a} \rho_{M}=-\sqrt{\frac{4 \pi G \omega}{3}} \rho_{M} \dot{\phi} .
$$

Unfortunately, it is not possible to determine what $A(\psi)$ must be to allow a mixed attractor. The root of this problem lies in the fact that $A(\psi)$ always appears in the combination $\zeta A(\psi) \dot{\psi}^{2}$ or its derivative, which means there are not enough equations to uniquely determine the time evolution of both $A(\psi)$ and $\dot{\psi}$. We may circumvent this problem, however, by a change of variables:

$$
\chi \equiv \int \sqrt{2 \zeta A(\psi)} d \psi
$$

The equations of motion become:

$$
\begin{array}{r}
\omega\left(\ddot{\phi}+3 \frac{\dot{a}}{a} \dot{\phi}\right)+\frac{\partial U(\chi, \phi)}{\partial \phi}=\sqrt{\frac{4 \pi G \omega}{3}}\left(\rho_{M}-e^{-\sqrt{\frac{16 \pi G \omega}{3}} \phi} \dot{\chi}^{2}\right) \\
\ddot{\chi}+3 \frac{\dot{a}}{a} \dot{\chi}+e^{\sqrt{\frac{16 \pi G \omega}{3}}} \frac{\partial U(\chi, \phi)}{\partial \chi}=-\sqrt{\frac{16 \pi G \omega}{3}} \dot{\chi} \dot{\phi}
\end{array}
$$

which must obey the constraints:

$$
\rho_{M}=\epsilon U(\chi, \phi)=\kappa \frac{1}{2} e^{-\sqrt{\frac{16 \pi G}{3}} \phi} \dot{\chi}^{2}=\gamma \frac{\omega}{2} \dot{\phi}^{2} .
$$

The total energy density evolves with the scale factor like

$$
\rho \sim a^{-\frac{3 \epsilon(2 \kappa+2 \gamma+\kappa \gamma)}{\kappa \gamma+\gamma \epsilon+\epsilon \kappa+\gamma \epsilon \kappa}}
$$

while the scale factor in turn evolves with time as

$$
a=a_{0}\left(\frac{t}{t_{0}}\right)^{\frac{2(\kappa \gamma+\gamma \epsilon+\epsilon \kappa+\gamma \epsilon \kappa)}{3 \epsilon(2 \kappa+2 \gamma+\kappa \gamma)}} .
$$

Thus the total energy density evolves with time as $\rho \sim t^{-2}$ just like in the single scalar case. Also analogous to the single scalar case, the field $\phi$ in the two-field case evolves with time as

$$
\phi=\phi_{0}+\frac{1}{\sqrt{\pi G \omega}} \ln \left[\left(\frac{t}{t_{0}}\right)^{ \pm \frac{1}{2 \mu}}\right]
$$


whereas the new field $\chi$ evolves as

$$
\chi=\chi_{0}\left(\frac{t}{t_{0}}\right)^{ \pm \frac{1}{\mu}}
$$

Here $\mu$ is a dimensionless ratio defined as:

$$
\mu=\frac{3 \sqrt{\epsilon}(2 \kappa+2 \gamma+\kappa \gamma)}{2 \sqrt{\kappa(\kappa \gamma+\gamma \epsilon+\epsilon \kappa+\gamma \epsilon \kappa)}}
$$

which is again similar to the single scalar case. Using this time dependence of $\phi$ in the dark matter conservation equation we get the constraint

$$
\frac{\gamma \kappa(\epsilon \kappa+\kappa \gamma+\epsilon \gamma+\epsilon \gamma \kappa)(3 \epsilon \gamma+3 \epsilon \kappa-3 \kappa \gamma \mp \sqrt{\epsilon \kappa(\epsilon \kappa+\kappa \gamma+\epsilon \gamma+\epsilon \gamma \kappa)})}{9 \epsilon^{2}(2 \kappa+2 \gamma+\gamma \kappa)}=0
$$

which is analogous to equation (53) relating $\xi$ to $\epsilon$ and $\kappa$ in the single scalar example. However, there is no variable $\xi$ in the two-scalar case (it is effectively set to $\frac{1}{2}$ ) so this equation acts as a constraint on $\epsilon, \kappa$, and $\gamma$ at the critical point rather than relating these variables to a model parameter. For the critical point to exist it must satisfy this constraint, but in order to be accelerated it must also have an equation of state satisfying

$$
w=\frac{\gamma \epsilon+\kappa \epsilon-\kappa \gamma}{\gamma \epsilon+\kappa \epsilon+\kappa \gamma+\kappa \epsilon \gamma}<-\frac{1}{3} .
$$

Unfortunately, the regions of $\{\kappa, \epsilon, \gamma\}$-space in which the constraint and the acceleration condition are satisfied do not intersect at any point. Thus, the hybrid model cannot both realistically model our universe and solve the coincidence problem.

\section{ASYMPTOTIC BEHAVIOR: GENERAL CASE}

Is there a deeper reason why the dual scalar model does not work but the single scalar model does? Certainly the failure of the former has nothing to do with the changes of variables since the same dark matter constraint and condition for acceleration result when we use $\psi$ instead of $\chi$ as well as when we use $\Omega^{2}$ instead of $\psi$. Furthermore, having more scalar fields (as in a Higher-Order Gravity model) does not help matters. To see this, let the Jordan frame action have a combined potential $\widetilde{U}\left(\psi_{0}, \ldots, \psi_{n-1}, \Omega^{2}\right)$ and a combined kinetic term $\widetilde{g}^{\mu \nu} \widetilde{K}_{\mu \nu}$ for the $\psi_{j}$. Note that we are not assuming any specific form for the individual 
kinetic terms save that they have two lower tensor indices. The individual terms may be multiplied by functions of the scalar fields (including $\Omega^{2}$ ), and they may even contain higher derivatives. The Einstein frame action in this example is

$$
\begin{aligned}
S= & \int d^{4} x\left\{\sqrt { - g } \left[\frac{R}{16 \pi G}-g^{\mu \nu} K_{\mu \nu}+U\left(\psi_{0}, \ldots, \psi_{n-1}, \Omega^{2}\right)\right.\right. \\
& \left.\left.-\frac{3}{32 \pi G} \Omega^{-4} g^{\mu \nu} \partial_{\mu} \Omega^{2} \partial_{\nu} \Omega^{2}\right]-L^{(M)}\right\}
\end{aligned}
$$

where $U\left(\psi_{0}, \ldots, \psi_{n-1}, \Omega^{2}\right)=\Omega^{-4} \widetilde{U}\left(\psi_{0}, \ldots, \psi_{n-1}, \Omega^{2}\right)$ and $K_{\mu \nu}=\Omega^{-2} \widetilde{K}_{\mu \nu}$. The Einstein frame scalar field stress-energy tensor is

$$
\begin{aligned}
T_{\mu \nu}\left(\psi_{0}, \ldots, \psi_{n-1}, \Omega^{2}\right)= & 2\left(K_{\mu \nu}-\frac{1}{2} g_{\mu \nu} g^{\alpha \beta} K_{\alpha \beta}\right)+g_{\mu \nu} U\left(\psi_{0}, \ldots, \psi_{n-1}, \Omega^{2}\right) \\
& +\frac{3}{32 \pi G} \Omega^{-4}\left(\partial_{\mu} \Omega^{2} \partial_{\nu} \Omega^{2}-\frac{1}{2} g_{\mu \nu} g^{\alpha \beta} \partial_{\alpha} \Omega^{2} \partial_{\beta} \Omega^{2}\right)
\end{aligned}
$$

and its conservation in an FRW cosmology yields

$$
\begin{aligned}
\frac{1}{2} \Omega^{-2} \frac{\partial \Omega^{2}}{\partial t} \rho_{M}= & \frac{\partial}{\partial t}\left(K_{00}+U\left(\psi_{0}, \ldots, \psi_{n-1}, \Omega^{2}\right)+\frac{3}{32 \pi G} \Omega^{-4}\left(\frac{\partial \Omega^{2}}{\partial t}\right)^{2}\right) \\
& +3 \frac{\dot{a}}{a}\left(2 K_{00}+\frac{3}{16 \pi G} \Omega^{-4}\left(\frac{\partial \Omega^{2}}{\partial t}\right)^{2}\right) .
\end{aligned}
$$

Here we have assumed that only the $0-0$ component of $K_{\mu \nu}$ is relevant to the homogeneous evolution, the other components entering the picture at level of first- or higher-order perturbations. This is an extremely generic assumption since there is no easy way to combine scalars into an object with two tensor indices besides including covariant derivatives, and only the 0-component of a covariant derivative contributes to the homogeneous evolution of the universe. One alternative would be to build $K_{\mu \nu}$ from the metric $g_{\mu \nu}$ multiplied by some function of the scalars; however, such an object could be re-interpreted as a potential term and would therefore not belong in $K_{\mu \nu}$ at all. Another possibility would be to use the four dimensional Levi-Civita density $\varepsilon_{\mu \nu \lambda \sigma}$ to mix up the indices of two contravariant derivatives, but doing so would always pair time derivatives with space derivatives so that every term 
would be negligible to zero-th order. Therefore let us assume that $K_{\mu \nu}$ is dominated by its 0-0 component.

To be on a critical point each scalar field must have a kinetic term either equal to zero or proportional to the energy density of dark matter. This implies that all non-vanishing kinetic terms must also be proportional to each other. Therefore, without loss of generality, we can treat $K_{\mu \nu}$ as a whole and require it to be proportional to the dark matter energy density. We now have

$$
\rho_{M}=\epsilon U(\psi, \phi)=\kappa K_{00}=\gamma \frac{3}{32 \pi G} \Omega^{-4}\left(\frac{\partial \Omega^{2}}{\partial t}\right)^{2},
$$

which is a generalization of equation (60).

If we follow a procedure similar to that used in Section XI for the dual scalar case we find that together these ratios along with the Friedmann equation and the equations of motion yield the exact same constraints on $\epsilon, \kappa$, and $\gamma$ as were found in equation (66). Furthermore, if the $0-0$ component of $K_{\mu \nu}$ is the only one relevant to the homogeneous evolution of the universe then the equation of state for this general kinetic term is the same as for a standard kinetic term, i.e., $w=1$. Therefore, the constraint that the overall equation of state be less than $-\frac{1}{3}$ is the same as the two-scalar case above, equation (67).

Thus having a kinetic term for $\Omega^{2}$ distinct from other kinetic terms for the $\psi_{j}$ prevents the model from solving the coincidence problem and also allowing accelerated expansion. But then why does the single scalar case work? It works because the field $\Omega^{2}$ is just a redefinition of the field $\psi$, which allows us to combine the kinetic terms in equation (14):

$$
L^{(\psi)}=\sqrt{-g}\left[\zeta A(\psi) \Omega^{-2}(\psi)+\frac{3}{32 \pi G} \Omega^{-4}\left(\frac{\partial \Omega^{2}}{\partial \psi}\right)^{2}\right] g^{\mu \nu} \partial_{\mu} \psi \partial_{\nu} \psi-\sqrt{-g} U(\psi)
$$

Let us refer to the quantity inside the brackets as $\frac{1}{2} \omega(\psi)$. Previously, we transformed this Lagrangian into one having a canonical kinetic term via the redefinition $\phi=\int d \psi \sqrt{\omega(\psi)}$. However, whether we choose to make this redefinition or not has no effect on the present discussion, and it is more instructive to use the general form. The scalar field equation of motion and the dark matter conservation equation are:

$$
\omega(\psi)\left(\ddot{\psi}+3 \frac{\dot{a}}{a} \psi\right)+\frac{1}{2} \frac{d \omega(\psi)}{d \psi} \dot{\psi}^{2}+\frac{d U(\psi)}{d \psi}=\Upsilon(\psi) \rho_{M}
$$




$$
\dot{\rho}_{M}+3 \frac{\dot{a}}{a} \rho_{M}=-\Upsilon(\psi) \dot{\psi} \rho_{M},
$$

and the condition for being on a critical point is

$$
\rho_{M}=\epsilon U(\psi)=\kappa \frac{\omega(\psi)}{2} \dot{\psi}^{2} .
$$

If we then follow the same procedure as before for finding $\rho_{M}(a)$ and $a(t)$ and then insert these into the dark energy conservation equation we find, instead of a constraint on the energy ratios or a relation between these and a model parameter, a more general constraint on $\omega(\psi)$ and $\Upsilon(\psi)$ :

$$
\frac{\kappa(\epsilon+\kappa+\epsilon \kappa)\left(3 \kappa-3 \epsilon \mp \frac{\sqrt{3} \Upsilon(\psi)}{\sqrt{4 \pi G \omega(\psi)}} \sqrt{\epsilon(\epsilon+\kappa+\epsilon \kappa)}\right)}{6 \epsilon^{2}(2+\kappa)}=0 .
$$

If $\kappa$ and $\epsilon+\kappa+\epsilon \kappa$ are non-zero then we have that the ratio

$$
\frac{\Upsilon(\psi)}{\sqrt{\frac{4 \pi G}{3} \omega(\psi)}}=\frac{\frac{1}{2} \Omega^{-2}(\psi) \frac{d \Omega^{2}(\psi)}{d \psi}}{\sqrt{\frac{8 \pi G}{3} \zeta A(\psi) \Omega^{-2}(\psi)+\left(\frac{1}{2} \Omega^{-2}(\psi) \frac{d \Omega^{2}(\psi)}{d \psi}\right)^{2}}}
$$

must be constant if the universe to is be on a mixed critical point. A comparison with equation (52) shows that this constant value is what we have been calling $2 \frac{\xi}{\sqrt{\omega}}$. The problem with the multiple scalar situation is that, since we cannot combine the kinetic terms for the $\psi_{j}$ and $\Omega^{2}$, the prefactor on the $\Omega^{2}$ kinetic term is just $\frac{3}{32 \pi G} \Omega^{-4}\left(\frac{\partial \Omega^{2}}{\partial \psi}\right)^{2}$ instead of the more complicated expression in equation (72). Thus, in the multiple scalar case we find that the constant value is actually just \pm 1 , and, as we shall soon show, there is no way to get an accelerated and mixed critical point with these values.

We have just seen that the condition of constant energy ratios enforces a relationship between the functions $\omega(\psi)$ and $\Upsilon(\psi)$; what about the other undetermined function, $U(\psi)$ ? It turns out that constant energy ratios also fix the form of $U(\psi)$, which is then related to $\omega(\psi)$ by

$$
U(\psi)=\frac{\rho_{M, 0}}{\epsilon} \exp \left\{\mu \sqrt{\frac{16 \pi G}{3}} \int_{\psi_{0}}^{\psi} \sqrt{\omega\left(\psi^{\prime}\right)} d \psi^{\prime}\right\} .
$$

Therefore, whenever the lone kinetic term is set to have constant positive coupling to gravity (via transformation or careful selection of $A(\psi)$ and $\Omega^{2}(\psi)$ ) the potential must be of exponential form in order for a mixed critical point to exist. 


\section{POSSIBLE GENERALIZATIONS}

In order for a universe exhibiting dark matter-dark energy coupling to evolve on a mixed critical point we have just argued that the potential must be related to the prefactor on the dark energy kinetic term; specifically, when the dark energy is minimally coupled we showed that the potential must be of exponential form. Let us assume this to be the case. Obviously a realistic universe does not evolve on the mixed critical point for all time; we require only an asymptotic approach to the point. This means that the requirement of an exponential potential is too tight. All that is required is an exponential tail that is energetically accessible from the rest of the potential.

For example, a potential formed from the sum of two exponentials (having parameters $\mu_{1}$ and $\mu_{2}$ ) will have two mixed, accelerated points for each one point in the single exponential case, i.e., one for each $\mu$. If the two $\mu$ s have the same sign the stable point will correspond to the potential term with the lower value of $|\mu|$ since that term will dominate on the tail. If the $\mu$ s have different signs the potential is U-shaped, and therefore the only stable point will be the one corresponding to the global minimum.

Another simple possibility is the potential derived from compactification of the KaluzaKlein extra dimension (see [26] for a discussion of this and other examples),

$$
U(\psi)=e^{-\psi / \sqrt{8 \pi G}}\left(1-e^{-\psi / \sqrt{8 \pi G}}\right)^{2}
$$

which has an exponential tail. This potential has another local minimum at the origin, though, so it has two possible late-time behaviors depending on $\dot{\psi}$. If $\dot{\psi}$ is below a certain threshold, the field oscillates about the origin, but if $\dot{\psi}$ is large enough, the field climbs the "hump" onto the exponential tail.

\section{CONCLUSIONS}

This article has discussed conformally transformed gravity theories and their possible use in solving the Coincidence Problem. Four types of gravity theories were examined. Depending on whether the original Jordan frame theory contained scalar fields, or functions, higher powers, or derivatives of the Ricci scalar, the resulting Einstein frame action involved scalar fields and a potential that could be completely, partial, or not at all determined. 
Indeed, there was found to be a degeneracy among Jordan frame theories in that there can be many with the same Einstein frame physics.

For a given Jordan frame theory there are several possible strategies for using a conformal transformation in a realistic cosmological scenario. The conformal transformation generates scalar fields in the Einstein frame that may be interpreted as cosmological quintessence, and it induces a coupling between these fields and the matter fields. There are a number ways of implementing this coupling depending on which matter fields are coupled, as well as which ones are present in the coupling term. The choice for the former need not imply the same choice for the latter as the Catalyzed Quintessence Model demonstrates. The physical interpretation of the conformal transformation itself can depend on these choices.

Many of the more complicated conformally transformed theories, i.e., those theories which contain more than one scalar field in the Einstein frame, were unable to solve the Coincidence Problem. Furthermore, for a single scalar field model to solve the Coincidence Problem there are constraints on the relationships between the scalar field coupling to gravity $\omega(\psi)$, the dark matter-dark energy coupling $\Upsilon(\psi)$, and the scalar potential $U(\psi)$ which must be obeyed. First, the quantity $\Upsilon(\psi) / \sqrt{\omega(\psi)}$ must equal some non-trivial constant or at least asymptotically approach one; second, the potential must have the form $U(\psi) \sim \exp \left\{\mu \sqrt{\frac{16 \pi G}{3}} \int_{\psi_{0}}^{\psi} \sqrt{\omega\left(\psi^{\prime}\right)} d \psi^{\prime}\right\}$ though perhaps only on the tail.

\section{ACKNOWLEDGEMENTS}

We thank H. Karwowski, C. Clemens, Y.J. Ng, H. van Dam, J. Engel, and K. Crooks for their comments. This work was supported in part by the U.S. Department of Energy under Grant No. DE-FG02-97ER-41036 
[1] I. Zlatev, L. Wang and P. J. Steinhardt, Phys. Rev. Lett. 82, 896 (1999). astro-ph/9807002.

[2] W. Zimdahl, D. Schwarz, A. B. Balakin and D. Pavon, Phys. Rev. D64, 063501 (2001). astro-ph/0009353

[3] C. Brans and R. H. Dicke, Phys. Rev. 124925 (1961).

[4] T. Damour, G. W. Gibbons and C. Gundlach, Phys. Rev. Lett. 64, 123 (1990).

[5] D. Tocchini-Valentini and L. Amendola, Phys. Rev. D65, 063508 (2002). astro-ph/0108143.

[6] L. Amendola and D. Tocchini-Valentini, Phys. Rev. D64,, 043509 (2001). astro-ph/0011243.

[7] R. Bean and J. Magueijo, Phys. Lett. B517, 177 (2001). astro-ph/0007199

[8] D. Torres, Phys. Rev. D66, 043522 (2002). astro-ph/0204504.

[9] V. Faraoni, E. Gunzig and P. Nardone, Fund. Cosmic Phys. 20, 121 (1999). gr-qc/9811047.

[10] R. Adler, M. Bazin and M. Schiffer, General Relativity. (1965). McGraw-Hill (New York), pages $260-80$.

[11] K. Maeda, Phys. Rev. D 393159 (1989).

[12] M. Carmeli, Classical Fields: General Relativity and Gauge Theory. (1982). J. Wiley (New York).

[13] G. Magnano and L. M. Sokolowski, Phys. Rev. D50, 5039 (1994). gr-qc/9312008.

[14] S. Gottlober, H.-J. Schmidt and A. A. Starobinsky, Class. Quantum Grav. 7, 893 (1990).

[15] J. D. Barrow and S. Cotsakis, Phys. Lett. B214 515 (1988).

[16] A. L. Berkin and K. Maeda, Phys. Rev. D44, 1691 (1991).

[17] K. Maeda, Phys. Rev. D37, 858 (1988).

[18] T. Futamase and K. Maeda, Phys. Rev. D39, 399 (1989).

[19] D. Wands, Class. Quant. Grav. 11, 269 (1994). gr-qc/9307034.

[20] H.-J. Schmidt, Class. Quantum Grav. 7, 1023 (1990).

[21] R. R. Caldwell, R. Dave and P. J. Steinhardt, Phys. Rev. Lett. 80, 1582 (1998). astro-ph/9708069

[22] R. D. Reasenberg, I. I. Shapiro, P. E. MacNeil, R. B. Goldstein and J. C. Breidenthal, Astrophys. J. Lett. 234, L219 (1979).

[23] L. Amendola, Phys. Rev. D62, 043511 (2000). astro-ph/9908023.

[24] R. R. Caldwell, Phys. Lett. B545, 23 (2002). astro-ph/9908168. 
[25] J. L. Crooks, PhD thesis. Department of Physics and Astronomy, The University of North Carolina at Chapel Hill. (2005).

[26] P. G. Ferreira and M. Joyce, Phys. Rev. D58, 023503 (1998). astro-ph/9711102. 